Revista Brasileira do Esporte Coletivo - v. 4. n. 1. 2020.

Artigo de Revisão

\title{
PRÁTICA ESPORTIVA + BOA ALIMENTAÇÃO = BENEFÍCIOS PARA A SAÚDE
}

${ }^{1}$ Bruno Freitas Santos

\section{RESUMO}

Introdução: O referido estudo apresenta-se como uma revisão de literatura. O mesmo trata da importância da alimentação e das atividades físicas, como um importante recurso para uma vida saudável e um aprendizado muito mais significativo, influenciando positivamente na saúde física e mental do sujeito. Objetivo: Buscou-se verificar que alimentação e as atividades físicas são ingredientes indispensáveis para um bom desempenho na saúde humana. Método: Este estudo está baseado em trabalhos teóricos (artigos e livros) de autores que discutem a temática, mostrando que as práticas físicas, seguida de uma boa alimentação podem e devem ser um recurso importante para a construção de uma vida ativa e saudável. Resultados e Discussão: Por meio da busca realizada, foi possível verificar que sem uma boa alimentação e sem atividades físicas, impossibilita-se a obtenção de uma saúde plena. Conclusão: Conclui-se que novas políticas públicas na área da alimentação e das práticas esportivas são imprescindíveis para a manutenção e obtenção da saúde pública.

Palavras-chave: Desenvolvimento, Nutrição, Saúde humana.

\section{SPORT PRACTICE + GOOD FOOD = HEALTH BENEFITS.}

\begin{abstract}
Introduction: This study is presented as a literature review. It deals the importance of food and physical activities, as an important resource for a healthy life and a much more meaningful learning, positively influencing the subject's physical and mental health. Objective: We tried to verify that food and physical activities are essential ingredients for a good performance in human health. Method: This study is based on theoretical works (articles and books) by authors who discuss the theme, showing that physical practices, followed by good nutrition can and should be an important resource for building an active and health life. Results and Discussion: Through the search, it was possible to verify that without a good diet and without physical activities, it is impossible to obtain full health. Conclusion: It is conclude that new public policies in the area of food and sports practices are essential for the maintenance and achievement of public health.
\end{abstract}

Keywords: Development, Nutrition, Human health.

1 Bruno Freitas Santos Discente do curso de Mestrado em Educação da Anne Sullivan University Graduado em letras pela Faculdade de Tecnologia e Ciências (FTC BA) Professor da rede municipal de ensino (BA) Endereço: Av. João Ribeiro do Vale n`950 - Qd. 05.4720-000 - Remanso/BA, Brasil.

E-mail: brunofreitas20017@.outloo.com.br

\section{INTRODUÇÃO}

A reflexão em torno das questões referentes à alimentação e as práticas esportivas, tem sido algo necessário e ao mesmo tempo preocupante, pois o número de pessoas, que se intoxicam com alimentos perigosos na forma sólida e liquida é crescente em todo o mundo. E que vivem de forma inativa é cada vez mais assustador. Sendo necessárias inúmeras ações e intervenções em prol de uma educação e reeducação alimentar, essas ações são de extrema relevância para todos os agentes envolvidos dentro do processo escolar. A nutrição é um campo da saúde de grande pertinência para todos. Sendo necessária a hidratação do 
corpo, através da ingestão de líquidos antes, durante e após a as práticas físicas, independente do peso corporal do sujeito.

Freitas e colaboradores (2007) explicam que, a melhor qualidade de vida dos indivíduos acontece por meio da prática de exercício físico, e de uma boa alimentação. 0 assunto sobre corpo e saúde, deve sempre atingir dimensões amplas e variadas. Afinal de contas, investir na saúde é um dos maiores investimentos que o homem pode realizar.

Vivemos numa era em que o correcorre da vida cotidiana, a vida agitada dos grandes centros urbanos, não permite que tenhamos uma alimentação saudável, e nos impende de ter uma pratica esportiva cotidiana. Recursos que, são aconselháveis para todos, porque os verdadeiros vilões da saúde humana são gigantescos para todos. Problemas dessa natureza são sempre crescentes, e sem sombras de dúvidas é um grande desafio, manter uma vida saudável e equilibrada.

Reconhecendo também, que o problema da má alimentação e dos hábitos errôneos na hora de se alimentar precisa de correções. É preciso, que haja lugares de referência de saúde, a ser seguido. A educação e a reeducação alimentar é uma grande lição de vida a ser aprendida e aplicada por todos, desde as crianças até os idosos. $E$ essa chamada educação e reeducação alimentar podem ser fortalecidas, através da frequência continua em academias. E de acordo a International Health, Racquet e Sportsclub Association (IHRSA, 2016) o cenário brasileiro, por exemplo, tem ocupado o segundo lugar no ranking mundial em número de academias, perdendo apenas para os Estados Unidos. Isso revela uma certa preocupação dos brasileiros não só com a estética corporal, mas também com a saúde no geral.

As questões alimentares e esportivas devem está sempre presente, em todas as instâncias da vida do sujeito, do qual o mesmo conseguirá optar por uma vida consciente frente aos benéficos ofertados pela boa alimentação e pelas práticas esportivas continuas. Desenvolver determinados hábitos, não é uma tarefa fácil, pois exige força de querer e muita determinação. Assim, criar e desenvolver estratégias, que começa pelo

\section{RESULTADOS E DISCUSSÃO}

As necessidades nutricionais são individuais. E todo e qualquer indivíduo precisa estar atento para todas essas carências e próprio eu. E isso é algo imprescindível. E de acordo com o Conselho Federal de Nutricionistas (CFN), através de sua Resolução CFN N600, 2018, reconhecer a necessidade da Nutrição em Esportes através de uma boa alimentação. $E$ assuntos pertinentes como esses devem fazer parte da realidade de todos. Afinal de conta, está nutrido é uma necessidade vital a todos os indivíduos.

Assim, o objetivo do presente estudo é analisar a relevância da alimentação e das atividades físicas, como um recurso auxiliar no processo da obtenção e manutenção da saúde pública. A justificativa, que impulsionou a elaboração desse artigo é a busca por ações, que tornem a vida mais significativa, para todas as fases da vida.

A estrutura desse trabalho se dá por meio de uma apresentação do posicionamento de alguns teóricos, acerca da temática, mostrando pontos convergentes e divergentes.

\section{MÉTODO}

Cervo, Bervian e Silva (2007, p.61), afirmam que a pesquisa bibliográfica se "constitui como um procedimento básico para os estudos e pesquisas científicas". Essa fase é crucial para o desenvolvimento de uma obra cientifica, é também um ponto de partida que permite a coleta e a construção das informações que estão em pauta.

O método aqui utilizado é o bibliográfico, que tem como principal característica "explorar por meio de diferentes autores a essência de um determinado assunto" (LAKATOS, 2007, p 107). Permitindo que, fosse construído passo a passo 0 referencial teórico desse trabalho.

Conforme foi apresentado na introdução, a pesquisa é uma revisão bibliográfica. Trata-se de uma pesquisa qualitativa, onde foram levantados diferentes posicionamentos acerca da temática, que auxilia em todas as fases da vida. Mostrando suas muitas vantagens, desde que seja consumida com cuidado e com controle, bem como os prejuízos dos alimentos ruins e da inatividade física. Quanto aos resultados, foram encontradas informações pertinentes para melhor fundamentar a referida pesquisa.

necessidades, independentemente do sexo, da idade, e estatura.

As questões relacionadas a alimentação têm um papel primordial, no que 


\section{ESPORTE, ALIMENTAÇÃO E SAÚDE.}

se refere a obtenção e manuıtıı̧au ua sauue pública. Mello, Luft e Meyer (2004) comentam que 0 Brasil tem demonstrado que em determinadas cidades brasileiras apresentam índices elevados de sobrepeso e obesidade em sua população infantil e jovem. E esses dados já são preocupantes, por uma falta de educação e reeducação alimentar, bem como a conscientização de práticas esportivas.

Para Balaban e Silva (2001) os porcentuais do sobrepeso são preocupantes e da obesidade é algo ainda maior. Essas requerem sérias intervenções emergenciais, para evitar uma série de problemas. Moraes, Oliveira e Fernandes (2008) trazem uma importante discussão sobre a obesidade nas crianças. Um grave problema que afeta 0 Brasil e o mundo; os pais carregam nas costas uma culpa nesses erros alimentares. Sendo necessário um estimulo para as atividades físicas em todos os ciclos da vida.

O sobrepeso e a obesidade são dois fatores de riscos, que geram uma série de consequências para a vida, para a sociedade, para a população num geral. Naghettini (2010) explica que, o acumulo excessivo de gordura, é fruto de uma série de erros alimentares, que começa desde muito cedo na primeira infância. Onde é obrigatório o estímulo de uma vida ativa e prática, sob a ótica dos esportes.

A má alimentação é prejudicada também pelo tabagismo, o que tem gerado a ociosidade, que é preenchida pela inatividade física. Desse modo, há uma necessidade de se reeducar hábitos e valores para ter uma vida ativa e saudável. A presença do sedentarismo é um grande vilão no século $X X I$, um fator de risco, que deve ser combatido através de boas práticas alimentares e de uma alimentação correta.

Atividades físicas exigem muito vigor e esforço físico dos indivíduos. Portanto, o desenvolvimento de hábitos saudáveis é uma necessidade do praticante, pois leveza, a resistência, a força, a agilidade e o domínio do corpo, dos músculos e movimentos requer um gasto de energia, que é reposto por meio da alimentação (SANTOS e KNIJNIK, 2009).

E os esportes são sempre sinônimos de pluralidade, que vem na forma de diferentes tipos de atividades físicas: como treinamentos, condicionamentos, atividades aeróbicas, atividades de força, atividades de flexibilidade, atividades de equilíbrio, atividades de lazer e uma atividade mais recente que é a ginástica laboral, um exercício físico orientado e praticado durante o horário do expediente, visando os muitos benefícios vala us iniluivilaiıos, mesmo em horário de trabalho.

Essa atividade quebra com as rotinas do sedentarismo, oferecendo uma vida melhor e saudável. Porque ao analisar os estudos de Uyeda e Toledo (2015) um quadro elevado de sedentarismo, pode causar quedas no rendimento no humor e um acréscimo de irritabilidade dos funcionários durante as suas respectivas funções.

A ingestão excessiva de alimentos ricos em gorduras e açúcares é um mal habito, que começa na adolescência e se perpassa por todas as fases da vida. Assim, a educação nutricional é uma base para uma vida plena e integral (DA SILVA VARGAS, 2011).

Neste contexto, a Organização Mundial de Saúde (2011) orienta que é importante desenvolver hábitos de alimentação saudável. $E$ isso deve começar desde a primeira infância, onde as crianças aprenderão a valorizar cada nutriente, presente em cada alimento. Esse hábito, servirá de suporte para a manutenção e obtenção da saúde, reduzindo consequentemente os risco de doenças, que todos os anos tem matado milhões de pessoas em todo o mundo (DE OLIVEIRA TEIXEIRA, 2010).

O Instituto Brasileiro de Geografia e Estatística (2011) expõe que houve um aumento do consumo de alimentos, que não são saudáveis, ricos em açúcares e gorduras. $E$ isso, reflete na balança, onde crianças, adolescentes e idosos estão bem acima do peso. Como solução é apontada, que os exercícios físicos, servem de apoio para ser paulatinamente uma vida mais ativa $e$ saudável. As questões referentes ao sobrepeso e a obesidade são dois graves problemas, que requer intervenções sérias e emergenciais.

Educar e reeducar no requisito alimentação são imprescindíveis. É aconselhável que, seja reduzido os gastos com alimentos gordurosos e açucarados, e investir maior tempo em atividades físicas continuas e permanentes. Substituindo os erros alimentares que foram aprendidos, desde a infância por uma nutrição completa e variada, com quantidade suficiente e de forma que atenda às necessidades do organismo humano, que sejam ricos em todos os nutrientes, tais como os carboidratos, proteínas, lipídios, vitaminas e minerais. Porque a ingestão de alimentos ricos nessa lista são os principais substratos para obtenção e manutenção da saúde humana, frentes as atividades em geral, sejam elas 
cotidianas ou as físici ESPORTE, ALIMENTAÇÃO E SAÚDE. il, o Ministério da Saúde COLABORADORES, 2014).

Figueiredo e colaboradores, (2011) explicam que 0 número de pessoas sedentárias é um grave problema que requer um conjunto de ações e de intervenções na forma de atividades físicas e uma alimentação equilibrada. A OMS (2006) recomenda que crianças não devam estar mais que uma ou duas horas em frente à TV, vídeo game, ou qualquer aparelho tecnológico diariamente. $\mathrm{O}$ tempo gasto, frente às tecnologias é um caminho para o sedentarismo, que tem causado uma série de problemas de diferentes naturezas.

O consumo de bebidas calóricas como refrigerantes tem sido uma grande preocupação no Brasil e no mundo. Os refrigerantes aparecem em sexto lugar na lista dos 20 alimentos mais consumidos por adolescentes (OMS, 2019). O consumo de frutas, leguminosas e vegetais é a receita infalível para uma saúde plena e integral do sujeito. $\mathrm{Na}$ alimentação a regra para esportistas, atletas ou pessoas comuns é que para manutenção de sua saúde é imprescindível que haja quantidades significativas de cobre, cromo, ferro, manganês, magnésio, sódio, zinco, cálcio, vitamina $A, E, C$ e vitaminas do complexo $B$, particularmente as vitaminas B6 e B12. Uma importante lista, que não pode faltar em nenhuma hipótese, quando se fala de promoção da saúde. E isso é muito descrito no guia alimentar, um documento de grande importância para todos, que sejam atletas ou não (BRASIL, 2003).

A Organização Mundial de Saúde (2006) realizou uma pesquisa com alguns países que possuem o maior número de obesos. Dentre eles aparecem, Índia, China e Brasil. No entanto o líder é os Estados Unidos com $70 \%$ de crescimento em três décadas. $E$ no mapa geral dos obesos em mais de 188 países, revelou que 1,6 bilhões de pessoas acima de 15 anos estariam com excesso de peso. $O$ que gera um número perigoso de obesos, um fator de risco que ameaça toda a sociedade, matando milhares de vida em todo o mundo. A Organização das Nações Unidas para a Alimentação e a Agricultura (FAO) divulgou recentemente o número de obesos que chega a (830 milhões) superando 0 número de famintos (820 milhões). A obesidade tem como principal vilão os alimentos ultra processados, que para muitos é algo prático e rápido, mas que traz prejuízos à saúde humana.

(८Uvo) apurila yue o crescimento é de $67 \%$ o número de obesos entre 2006 e 2018, a maior taxa em 13 anos. Desse modo, a implantação de políticas públicas na forma de atividades físicas é uma solução para amenizar esses graves problemas, que são de saúde pública.

A realização de atividades físicas é sempre sinônimo de vida ativa. Dessa forma a frequência e a duração dos diferentes tipos de atividades como caminhadas moderadas é sempre um bom caminho para obter a saúde física e mental.

De acordo com Chiara e Sichieri, (2001) o consumo de gordura trans, é um grande perigo para a saúde pública. $\mathrm{O}$ recomendável pela OMS (2011) é que todo ser humano alimente-se de pelo menos a ingestão diária de 2.500 calorias ao dia. O excesso de tudo isso, gera uma série de problemas, que vai pouco a pouco vai sendo acumulado, sendo $o$ princípio de um sobrepeso e possivelmente de uma obesidade. É importante ressaltar, que esse valor pode variar de acordo com as necessidades, que cada indivíduo tem diariamente.

$\mathrm{Na}$ concepção de Pioltine, Paternez e Morimoto (2013) o consumo excessivo de gorduras totais, saturadas e colesterol ruim tem levado muitos a quadro caóticos de saúde, que começam mascarados e de forma sutil, sendo necessária a substituição de uma alimentação baseada no consumo regular de frutas, verduras e legumes, na qual ajudam na prevenção e diminuição do risco cardiovascular e excesso de peso.

Alimentos industrializados são os grandes inimigos da alimentação dos brasileiros, pois os mesmos são ricos em gorduras ruins, o que é muito prejudicial à saúde humana, o que tem contribuído para o aumento da obesidade e de doenças cardiovasculares. A OMS (2006) recomenda que os indivíduos adotem as atividades físicas contínuas, durante toda a vida, pois é reduzido o risco de doenças cardiovasculares, que todos os anos fazem milhares de óbitos em todo mundo.

A saúde necessita de uma atenção especial dos gestores e de todas as equipes que trabalham diretamente ou indiretamente com saúde. A implementação de ações, sob o viés do estímulo à promoção de um estilo de vida saudável (alimentação e atividade física). A ingestão inadequada de alimentos, não proporciona o estímulo suficiente para um bom desempenho físico, além de ser o primeiro passo para o surgimento de várias outras doenças, que são silenciosas e perigosas. E 
esse assunto é também d€ ESPORTE, ALIMENTAÇÃo E SAÚDE. es, que precisam caminhar Araújo (2004), explica que ııuıus uus ılıaıs dos indivíduos, que são acometidos na área da saúde pública, são perigosas armadilhas, que o próprio homem constrói e ingere por meio da sua própria alimentação.

Desencadeado um corpo com um grande índice de excesso de gordura abdominal, o que é um risco para os demais órgãos humanos. A quantidade de gordura corporal ideal no homem pode variar entre 16 e $20 \%$ e na mulher entre 20 e $24 \%$, o que passar disso gera um sobrepeso e segue para a obesidade propriamente dita. A qualidade alimentar de tudo, que comemos é um fator decisivo, quando almejamos saúde e qualidade (MOURA E COLABORADORES, 2015).

O excesso de peso, na sociedade contemporânea é um grave problema que precisa ser substituído por hábitos saudáveis. Evitando os alimentos que se apresentaram como marcadores de risco de doença cardiovascular, como aqueles que são ricos em gorduras e sódio. Os riscos de mortalidade podem ser evitados, desde que alguns alimentos sejam evitados e substituídos por práticas esportivas contínuas (ROMANZINI, PELEGRINI E PETROSKI, 2011).

Segundo a Organização Mundial de

elll culluvinalicia.

Para Da Silva e colaboradores (2012) a associação do exercício físico com a alimentação equilibrada é a maior e a melhor dieta para todas as fases da vida. E quando é realizada com disciplina e continuidade os resultados são certos. Assim, é recomendável pela OMS (2006), que cada indivíduo adote essa postura.

O comportamento sedentário vem na forma de muito tempo gasto em televisão, computador, videogame e outros eletrônicos. O que torna as pessoas preguiçosas para o exercício contínuo das atividades físicas. As intervenções precisam ser tomadas, quando se trata da prevenção e da promoção da saúde humana, pois ela é o maior tesouro que podemos desfrutar. De acordo com Hallal et al., (2012) a falta de práticas físicas regulares está associada a uma série de doenças cardiovasculares, às diabetes mellitus tipo 2 , à obesidade, a alguns tipos de cancro. Doenças que são fatores de riscos, e que servem de ponte para o surgimento de várias outras doenças.

Neste sentido, a evidência da eficácia da atividade física é inquestionável, quando se fala de benefícios para a saúde. Desse modo, a necessidade de incorporação um novo estilo de vida ativa, tornou-se imprescindíveis a todos, que almejam obter benefícios de uma saúde plena e permanente (FARIAS JÚNIOR, 2011).

Pensar em saúde é pensar em qualidade de vida, e pensar em qualidade de vida é pensar em alimentação versus esportes. Uma relação que é construída, através da harmonia entre os indivíduos, proporcionando um modo de viver pleno e sustentável. A proposta de uma vida saudável é capaz de transformar toda uma realidade, possibilitando o acesso à promoção e a obtenção da saúde. Os benefícios para a saúde podem ser contemplados através de programas vigorosos de atividade física e de alimentos amigos da prevenção, obtenção e manutenção da saúde (WORLD HEALTH REPORT, 2002). para a prevenção da saúde humana, dentre

(WORLD HEALTH REPORT, 2002).




\section{CONCLUSÃO}

Percebe-se, que as práticas físicas e uma boa alimentação são de fundamental importância para a qualidade de vida e para a longevidade, uma receita infalível que até hoje tem grande relevância para todos os públicos de todas as idades. Benefícios esses, que já foram comprovados e revistos por meio de estudos, onde seres humanos são amostras vivas de inúmeras investigações.

Assim, as atividades físicas e uma boa alimentação aparecem como excelentes antídotos no combate ao controle de doenças crônicas, como a hipertensão, obesidade, depressão e diabetes. Uma vida saudável, nem sempre depende de um alto custo, na maioria das vezes depende de atitudes comprometedoras e simples, como usar o espaço de sua sala e se exercitar periodicamente e alimentos simples, que podem ser comprados com baixo custo como os ovos ricos em proteínas, vitaminas e minerais.

Hábitos saudáveis como estes precisam ser paulatinamente conquistados e praticados por todos, independentemente da idade, sexo, classe social, pois o bem estar é necessário a todos. $\mathrm{E}$ a maior riqueza que se pode conquistar é a saúde emocional, física e mental. E só pode, ser construído através de uma adequada alimentação versus atividades físicas (esportes).

Por fim, em resposta ao objetivo proposto, foi possível refletir sobre a temática construindo uma visão esclarecedora sobre a manutenção da saúde de maneira geral. Sugerem-se outros estudos dentro da temática em questão, para que sejam aprofundados pontos tão importantes como prática esportiva e alimentação.

\section{REFERÊNCIAS}

BALABAN, G.; SILVA, G. Prevalência de sobrepeso e obesidade em crianças e adolescentes de uma escola da rede privada de Recife. Jornal de Pediatria, v. 77, n. 2, p. 96-100, 2001.

BRASIL. Agência Nacional de Vigilância Sanitária (Anvisa). Brasília, [2003?]. Disponível em:<http://www.anvisa.gov.br/divulga/noticias/2003>. Acesso em: 13 jun. 2019.

BRASIL. Ministério da Saúde. Secretaria de Vigilância em Saúde. Secretaria de Atenção à Saúde. Diretrizes e recomendações para o cuidado integral de doenças crônicas não-transmissíveis. Brasília: Secretaria de Vigilância em Saúde/Secretaria de Atenção à Saúde; 2008. (Série Pactos pela Saúde 2006).

CERVO, A. L.; BERVIAN, P. A.; SILVA, R. Metodologia científica. 6. ed. São Paulo: Pearson Prentice Hall, 2007.

CHIARA, V. L.; SICHIERI, R. Consumo alimentar em adolescentes. Questionário simplificado para avaliação de risco cardiovascular. Arquivo Brasileiro de Cardiologia, v. 77, n. 4. p. 332-336, 2001.

CONSELHO FEDERAL DE NUTRICIONISTAS. Inserção Profissional dos Nutricionistas no Brasil. CFN. Brasília. 2018.

DA SILVA VARGAS, I. C.; e colaboradores. Avaliação de programa de prevenção de obesidade em adolescentes de escolas públicas. Revista Saúde Pública, v. 45, n. 1, p. 59-68, 2011.

FREITAS, S. M.; SANTIAGO, S.; VIANA, T.; LEÃO, A. C.; FREYRE, C. Aspectos motivacionais que influenciam a adesão e manutenção de idosos a programas de exercícios físicos. Revista Brasileira de Cineantropometria e Desempenho Humano. Florianópolis, v. 9, n. 1, p. 92-100, 2007.

IHRSA Global Report. Global Health Club Industry Posts Growth. International Health, Racquet \& Sports club Association, p. 21-26, 2016.

FARIAS JÚNIOR, J. C. (In) ESPORTE, ALIMENTAÇÃO E SAÚDE. ntário: estamos caminhando para uma mudança de paraaıgma! Kevısta brasııeıra de Atıvıdaae rısıca \& Saúde, v. 16, n. 4, p. 279 280. 2011.

FORTES, L. S.; ALMEIDA, S. S.; FERREIRA, M. E. C. Insatisfação corporal e comportamento alimentar inadequado em jovens futebolistas. Avaliação Psicológica, v. 14, n. 2, p.179-187, 2015. 
FIGUEIREDO, C.; e colaboradores. Obesidade e sobrepeso em adolescentes: relação com atividade física, aptidão física, maturação biológica e "status" socioeconômico. Revista Brasileira de Educação Física e Esporte, v. 25, n. 2, p. 225-235, 2011.

hallaL, P. C., BAUMAN, A. E., HEATH, G. W., KOHL, H. W., LEE, I.-M., \& PRATT, M. (2012). Physical activity: more of the same is not enough. Lancet, n. 380, p. 190-191, 2012.

INDICADORES sociais mundiais: uma análise dos resultados da alimentação equilibrada 2010. Rio de Janeiro: IBGE, 2011. 151 p. Acompanha 1 CD- ROM. (Estudos e pesquisas. Informação demográfica e socioeconômica, n. 28). Disponível em: <http://www.ibge.gov.br/ home/estatistica/populacao/alimentacao2010/indicadoressociais/indicadores_sociais_mundiais.pdf $>$. Acesso em: fev. 2019.

LAKATOS, E. M.; MARCONI, M. A. Fundamentos de metodologia científica. 5.ed. São Paulo: Atlas, 2003.

MELLO, E. D.; LUFT, V. C.; MEYER, F. Childhood obesity: towards effectiveness. Jornal de pediatria, v. 80, n. 3, p.173-182, 2004.

MORAES, A. C. F.; OLIVEIRA, H. G.; FERNANDES, C. A. M. Prevalência de sobrepeso e obesidade em alunos do ensino fundamental da cidade de Maringá - PR. Ciência, Cuidado e Saúde, v. 6, n. 2 supl., p. 364-369, 2008.

MOURA, U. I. S.; MENDES, L. R.; SILVA, I. P. O.; ÂNGELO, R. C. O.; SCHWINGEL, P. A. Consumo alimentar, perfil antropométrico e imagem corporal de bailarinos clássicos do Vale São Francisco. Revista Brasileira de Nutrição Esportiva. São Paulo, v. 9. n. 51. p. 237-246, 2015.

NAGHETTINI, A. V.; e colaboradores. Avaliação dos fatores de risco e proteção associados à elevação da pressão arterial em crianças. Arquivo Brasileiro de Cardiologia, v. 94, n. 4, p. 486-491, 2010.

UYEDA, M.; TOLEDO, L. F. S. Comportamento alimentar de atletas no pré treino de uma cidade do interior do estado de São Paulo. Saúde em Foco, 2015.

ORGANIZAÇÃO MUNDIAL DE SAÚDE (OMS). Conferência mundial sobre os determinantes sociais da saúde. Diminuindo as diferenças: a prática das políticas sobre os determinantes sociais de saúde: documento para discussão. Rio de Janeiro, out. 2011. Disponívelem:<http://www.who.int/sdhconference/discussion_paper/Discussion_Paper_PT.pdf>. Acesso em: 29 mar. 2019.

ORGANIZAÇÃO MUNDIAL DE SAÚDE (OMS). Grupo de Estudo Multicêntrico de Referência da saúde pública Crescimento. Normas de desenvolvimento saudável da OMS: Comprimento/altura por idade, peso por idade, peso-por-comprimento, peso por altura e índice de massa corporal por idade: Métodos e desenvolvimento. Genebra, OMS, 2006.

PINTO, S. L.: e colaboradores. Prevalência de pré-hipertensão e de hipertensão arterial e avaliação de fatores associados em crianças e adolescentes de escolas públicas de Salvador, Bahia, Brasil. Caderno de Saúde Pública, v. 27, v. 6, p.1065-1076, 2011.

PIOLTINE, M. B.; PATERNEZ, A. C. A. C.; MORIMOTO, J. M. Perfil dietético e risco cardiovascular em adolescentes de uma escola privada de São Paulo. Ciência \& Saúde, n. 6, n. 3. p. 149-156, 2013.

ROMANZINI, M.; PELEGRINI, A.; PETROSKI, E. L. Prevalência e fatores associados à obesidade abdominal em adolescentes. Revista Paulista de Pediatria, v. 29, n. 4, p. 546-552, 2011.

SANTOS, S. C.; KNIJNIK, J ESPORTE, ALIMENTAÇÃO E SAÚDE. intermediária. Revista Mackenzie de Educação Física e Ėsporte, v. 5, n. 1, 2009.

SILVA, G. G. S. et al. Momento dedicado à espera e à promoção da saúde. Psicologia: ciência e profissão, Uberlândia, v. 33, n. 4, p. 1000-1013, 2012. 
ESPORTE, ALIMENTAÇÃO E SAÚDE.

STOTZ, E. N.; ARAÚJO, J. W. G. Promoção da saúde e cultura política: a reconstrução do consenso. Saúde sociedade. São Paulo, v. 13, n. 2, p. 5-19, ago. 2004.

WORLD HEALTH REPORT 2002: Reducing risks, promoting healthy life. Geneva, World Health Organization, 
